\title{
XXIX. ON THE THEORY OF GELS.
}

\section{By SAMUEL CLEMENT BRADFORD.}

(Received September 10th, 1918.).

IN the present paper it is proposed to develop the theory, previously outlined $[1917,1,2]$, that the gelation of the natural emulsoids is a crystallisation process, which follows from von Weimarn's theory in consequence of their small coefficient of diffusion and the considerable viscosity and molecular aggregation of their sols. The properties of gels result simply from the structure thus involved. In certain cases, such as the so-called thermal anomaly of gels, and the heat of imbibition of gelatin, where the recorded facts are at variance with the theory, previous observations have been shown to be in error and results obtained in agreement with the values required.

\section{ON THE REVERSIBLE SOL-GEL TRANSFORMation.}

Perhaps the theory of gel structure which has found most favour of late is that adopted by W. Ostwald which considers gels to be composed of two liquid phases persisting from the sol stage. Hatschek's mathematical investigation [1917] showed, from the stress-elongation curve, that this hypothesis is untenable. The assumption is implied that gels have a solid disperse phase, consisting of discrete particles, or of aggregations of such particles. We thus return to the ideas comprehended in the earliest theories of gel structure. M. L. Frankenheim [1835] considered that gels were composed of aggregates of small crystals with pores between, which might account for the low density, easy solubility and elasticity of such bodies. K. von Nâgeli [1858] thought that a distinction should be made between distensible substances like gels and merely porous bodies, He supposed that gels consisted of molecular complexes or micellae with crystalline properties, separated by skins of water and forming meshes (or interstices) in which the water was contained by molecular attraction. Later $[1862,1]$ he wrote "Organised substances are composed of crystalline doubly refractive molecules aggregated from many atoms but which lie closely in definite arrangement with one another. In the moist condition, in consequence of the unbalinced attraction, each is 
surrounded with a skin of water." It has recently been suggested by more than one writer that this theory agrees most nearly with the properties of gels, and further study fully confirms this view. Although von Nägeli used the term "meshes (Maschen)" he may have referred merely to the interstices in the micellae. This appears probable in the light of a quotation given below. However, from this time, a number of unsuccessful attempts have been made to devise a geometrical framework which would account for the properties of gels. O. Bütschli's extensive researches $[1892 ; 1896,1898,1900]$ on foams and gel structure are well known. As a result of microscopic examination he came to the opinion that the properties of gels might be explained upon the basis of a honeycomb structure in which the walls were permeable to liquids because of their extreme thinness, although he admitted that the walls might be porous.

W. Pauli [1902] objected to Bütschli's assumption that the use of alcohol and chromic acid in his experiments to make the microstructure visible did not alter the structure of the gels. Von Weimarn [1914] also pointed out that the honeycomb appearance is probably an optical effect due to the overlapping of grains. From a study of imbibition Arisz [1915] came to a decision adverse to a honeycomb structure.

Moreover, if such a structure is dependent on the directive forces of crystallisation, a different arrangement would seem to be implied for each gel. Such differences in the structure of microscopically visible crystal aggregates were indeed found by Zsigmondy and Bachmann [1912] in their work on the gelation of sodium and potassium oleate, palmitate and stearate sols. It seems probable therefore that a single network will not account for the elastic and thermal properties of different gels.

The hypothesis of a network is not supported by the viscous behaviour of gels which gradually mould themselves to the shape of the containing vessel. The gradual motion of small solid masses through gels, under the influence of gravity or of diffusing streams of particles, leaving the gel apparently unchanged, has frequently been noticed during recent work. Moreover suspended cylinders tended to become ellipsoidal.

Further evidence against a net structure is afforded by the lens-shaped form of gas bubbles generated in gels. This phenomenon was investigated by Hatschek [1914] who found that 'the bubbles place themselves at right angles to pressure and parallel to tension applied to the gel. By measuring the angles between pairs of bubbles in 10 per cent. gelatin, Hatschek found certain values approximately repeated a number of times, although other 
measurements varied enormously. A similar appearance may be obtained in 1 per cent. agar by immersing the free gel containing $N / 5 \mathrm{Na}_{2} \mathrm{CO}_{3}$ in $\mathrm{N} / 4 \mathrm{CH}_{3} \mathrm{CO}_{2} \mathrm{H}$. Planes of cleavage are clearly suggested. The simplest structure that would conform approximately to the conditions is that of piled shot. This agrees with the globulitic structure observed ultramicroscopically by Bachmann [1911]. Examination of gelatin gels of from 1-6 per cent., from which the liquid had been expressed, showed irregular flocks of separate granules with clear spaces intervening. Increasing concentration reduced the size of the grains (as would be required by von Weimarn's theory) and, at the same time, the empty spaces gradually disappeared, until, at sbout 6 per cent., the solid phase filled the whole field and the single granules could be no longer differentiated. Agar and silicic acid gels showed a similar globulitic structure.

From these considerations it seems probable that the solid phase of gels consists of discrete granular particles. Since it is now recognised that the distinction between the suspensoids and emulsoids is rather a question of the affinity between the two phases than of the solid or liquid state of the disperse phase, there seems to be the less reason for refusing to extend von Weimarn's theory to the natural emulsoids and regard their gelation as a crystallisation process.

A connection between the precipitation of colloids and the process of crystallisation is certainly indicated by the fact that both suspensoids and emulsoids exhibit the phenomenon of supersaturation. W. Ostwald [1897] has shown that there is a minimum quantity of a substance which is capable of causing the crystallisation of a metastable solution. In the case of sodium chlorate this is equal to about $10^{-10} \mathrm{~g}$., corresponding to a cube with a side of several microns. This is well above the size of the disperse phase in sols. The existence of sols of greater concentration than the ordinary solubility of the substance concerned is therefore in harmony with the theory of supersaturation. The phenomena of supersaturation have frequently been observed in the case of sols. Thus gold separates spontaneously from gold sols of more than a certain concentration and the precipitation may be accelerated by adding a few drops of a second solution of colloid gold. Garrett [1903] found that gelatin sols gelate more rapidly if some solid gelatin, prepared by rapidly cooling the same sol, be added. The spontaneous coagulation of rubber emulsions is hastened by the addition of solid rubber. Additional evidence is furnished by the temperature variation of the concentration of the liquid expressed from gels. In the liquid from a 2.3 per cent. agar gel 
Hardy [1900] found from 0.09 per cent. of agar at $5^{\circ}$ to 0.47 per cent. at $36^{\circ}$. This is corroborated by the influence of concentration on the temperature of gelation [Pauli and Rona, 1902; Rohloff, 1907; Levites, 1908].

The literature of organic chemistry abounds in descriptions of definite substances which occur in the gel form. Frequently, by suitable choice of solvent, these have also been obtained as well defined crystals. The following examples are taken at random. The sodium salt of acrylic acid occurs as microscopic lenticular needles. The boiling saturated aqueous solution sets on cooling to a jelly which shows no structure under the microscope [Linnemann, 1874]. Glycyrrhizic acid, $\mathrm{C}_{41} \mathrm{H}_{63} \mathrm{O}_{18} \mathrm{~N}$, which occurs in licorice root, crystallises from alcohol as light yellow glistening leaflets, which are the better formed the slower the separation from alcohol. It is characteristically little soluble in water at ordinary temperatures. In boiling water the substance is easily soluble and the solution forms on cooling a light wine coloured jelly. By mixing $1 \mathrm{~g}$. of glycyrrhizic acid with $100 \mathrm{cc}$. of water a jelly is formed, after some hours standing, of such a consistency that the vessel may be inverted without loss [Habermann, 1879].

The gels of camphorylphenylthiosemicarbazide, investigated by Hatschek [1912], led him to suspect a crystalline structure formed by growth of amicrons into a network: a conclusion which he hesitated to accept on account of the mathematical difficulties. These gels appear to be typically elastic, and are produced (1) when a hot alcoholic solution of the substance up to about 5 per cent. is suddenly cooled (2) from the gradual or rapid cooling of toluene solutions of similar concentration, or (3) by pouring an alcoholic solution into petroleum. The latter method gives gels with a concentration as small as $1: 400$. The solubilities in alcohol, toluene and petroleum are respectively 0.5 per cent., 1 per cent. and very small. Von Weimarn's theory affords a ready explanation of such cases. From the examination of a very large number of precipitates von Weimarn showed that the degree of dispersity in which a substance occurs is regulated by the number of crystallisation centres, $N$, produced according to the formula $N=K P / L$, where $L$ is the solubility of the substance, $P$ the excess concentration $(C-L)$, and $K$ is a factor which depends on the degree of aggregation of the reaction components in solution and the viscosity of the reaction medium. A sol or gel is formed according as a large value of $N$ is produced from a small value of $L$ or a large value of $P$. Intermediate values of $L$ and $P$ give rise to micro or macro crystals. By actual inoculation of supersaturated solutions, von Weimarn has shown that in every stage, except the two extremes, the solid phase is crystalline. 
He infers that the disperse phase of such sols and gels is likewise crystalline though the particles are too small to act as nuclei.

Considering the general case of a substance of high molecular weight and very low diffusivity, let this be treated with a liquid in which its solubility, $L$, shall be smail in the cold and increase considerably, but not too rapidly on heating. Then, on applying heat, the process will be reversible and the Noyes-Nernst formula for the velocity of solution, $\nabla=\frac{D}{\delta} S(L-C)$, will hold. Since $L$ is very small, $V$ will be very small indeed, and the sol stage will persist. Further heating will lead to the gradual breaking up of the aggregated particles of the sol and an approach to true solution. If the stage of single molecules is reached, this may be regarded as a true solution, although the diffusion constant will be that of a sol. Notwithstanding the size of the molecules, the system may be looked upon as homogeneous and von Weimarn's formula applied. On cooling, with reduction of $L$, aggregation will commence, but the velocity of crystallisation, $V=\frac{D}{\delta} S(C-L)$, will be a minimum, so that diminution of $L$ will give a larger and larger $P$ and a still greater value of $N$, since $\frac{d N}{d L}=-K \frac{C}{L^{2}}$. The velocity of crystallisation will also increase, and, if the laws of, supersaturation apply, spontaneous crystallisation will eventually take place. Since $N$ must necessarily be very large, from the large values of $P$ and $K$, as well as the small value of $L$, a gel will be formed. Owing to the small velocity of crystallisation, gelation will however take place slowly. The formation of a gel will therefore be dependent on the concentration, the thermal variation of solubility and the factor $K$, which represents the degree of association and viscosity of the sol.

The natural emulsoids such as gelatin and agar appear to differ from the organic substances considered above, merely in the fact that they do not ordinarily occur in the crystalline form, though von Weimarn [1910] indeed claims to have obtained crystals of gelatin. Although little is known as to the true solubilities of these substances, there seems to be no reason for regarding them as exceptions to the general case. Actually their known properties are exactly such as to bring them within its scope. They form sols of considerable concentration, aggregation and viscosity. Heating at constant temperature decreases their viscosity, while cooling increases it [Levites, 1908], changes which are due to variation in the number as well as the size of the disperse particles and are thus seen to be in accordance with Einstein's formula. Osmotic pressure varies in the opposite sense.

The heat coagulation of albumin may be explained on the supposition of 
the chemical production of an insoluble compound. In this case the gelation should be, and actually is, irreversible. Silicic acid behaves very much in the same way as the typical case of aluminium hydroxide investigated by von Weimarn. The gel is formed immediately when the equivalent amount of hydrochloric acid is mixed with a moderately concentrated solution of potassium silicate. This is due to the sudden production of a highly aggregated insoluble form of silicic acid, owing to the concentration, aggregation and viscosity of the reaction medium. When, however, the silicate solution is slowly stirred into the mineral acid, thus keeping down the value of $K$, probably a true solution of silicic acid is formed at first, as is indicated by its high diffusivity.

While the foregoing cannot be considered as a rigid proof of the hypothesis, it seems permissible, in the absence of evidence to the contrary, to regard the reversible sol-gel transformation of the natural emulsoids as due to their large value of $K$, together with their low diffusion constant, which, by retarding crystallisation, allows the accumulation of a sufficiently large excess concentration to cause gelation on cooling. The theory receives confirmation from the fact that all the properties of gels are found to be consistent with the structure thus conditioned, and from the verification of certain new facts indicated thereby.

Similar considerations would appear to account for the occurrence also of resins and of glass. Indeed, from experiments on the introduction of metals into the surface layer of glass, Rhemberg is convinced that we should regard this as a substance full of ultramicroscopic pores. Various definite organic substances are easily obtained in either the crystalline or resinous form at will. Piperine crystallises from alcohol in monoclinic prisms which melt at $132^{\circ}$ and set on cooling to a light yellow transparent resin. On heating to $100^{\circ}$ the resin loses its transparency, becoming nearly opaque, like sulphur, owing to its transformation into a mass of microscopic crystals which undergo no further change until they melt at $132^{\circ}$. On cooling the resin is formed as before. If this is kept for about ten days, yellow sulphurlike spots of microscopic crystals begin to appear and very gradually extend throughout the mass. But after heating to $180^{\circ}$ for an hour, this change does not begin in $2 \frac{1}{2}$ years [Madan, 1901]. 


\section{ON SPHERITES IN RELATION TO GEL STRUCTURE.}

Before proceeding to the examination of the properties of gels, it seems worth while to inquire into the actual form of the disperse phase. As mentioned above, ultramicroscopic evidence shows a globulitic structure, but since the specific surface of gels must be extremely large, it seems probable that the individual grains are themselves aggregated. Von Nägeli was unable to say whether his micellae were, identical with spherites', but the evidence now available points to this conclusion. W. Ostwald [1891] states that microscopic observation of the process of crystallisation shows that (at least in the case of substances of low melting point) small spherical bodies first appesr. Only after they have attained a certain size do they assume crystalline shape. Linck [1839] first observed that substances deposited from solution appear originally as small droplets. Frankenheim [1860] describes the appearance of globules in a drop of saturated aluminium sulphate in water made turbid by addition of alcohol. However, Richards and Archibald's cinematogräphic study [1901] of the first appearance of growing crystals failed to show the occurrence of globules in any of the cases investigated, and it is doubtful whether a transitory spherical.phase appears, except in the case of substances of low melting point.

Spherites were first studied by Brewster [1853] who observed their production when solutions were allowed to crystallise under pressure between glass plates. He believed the pressure to be an essential cause of the phenomenon, but noticed the contributing effects of quick cooling and increase of viscosity. Rainey [1858] obtained microscopic spherites of calcite by allowing the slow mixing by diffusion of solutions of $\mathrm{CaCl}_{2}$ and $\mathrm{Na}_{2} \mathrm{CO}_{3}$ in gum water. Kuhlmann, in 1863, pointed out the important influence of in creasing the viscosity of the solution. With a view to slowing the crystallisa tion, he added such substances as gum and gelatin. When crystallisation took place sufficiently rapidly, the crystal particles which first appeared quickly grew into spherites. The structure of such bodies, occurring naturally in Acetabularia, was described by von Nägeli $[1862,2]$. "The smallest spherites (up to about $40 \mu$ ) were perfectly spherical... .In the unchanged state...they appear almost like oil drops or starch grains, though more glass like.... Often they show clear banding. The bands have a very regular and quite concentric relation to the surface... This stratified arrangement proves that the spherites

1 In this paper the term "spherite" is used to include spherical aggregates, spherical crystals, spherocrystals, spherulites, globulites and all such approximately spherical crystalline aggregations, the origin and structure of which appears to be similar. 
grow by additions to their surface... They appear as clear stripes which are mostly at exactly equal distances apart. Besides the concentric bands, frequently there appears a radial striation... .The substance is divided thereby into meshes of more or less quadratic form.... The ringed structure of the spherites is very like that of starch grains and cell membranes... By drying they become dark, since all their tiny meshes become filled with air." Von Nägeli also observed the beautiful brushes and rings which these crystal aggregates show in polarised light. Frankenheim remarks on the periodic growth of the spherites, which he considens the cause of the concentric rings like the yearly rings observed in a sawn tree stem. "After a time the process becomes slower, sometimes part will redissolve, but soon the exhausted liquid recovers itself, and growth begins anew, soon again to be interrupted." Frankenheim also recognised the relation between the concentric zonal structure frequently observed in minerals and the periodic growth of spherites. Dallas [1875] obtained beautiful spherites, especially of copper sulphate, lead acetate and morphine hydrochloride, by spreading the solution on glass plates and allowing rapid evaporation. Vogelsang [1875] studied the crystallisation of sulphur in carbon disulphide containing thickening material. The substance first appeared in the form of supersaturated droplets as Frankenheim had observed. These gave up more and more of the contained liquid and gradually solidified to isotropic spheres, or spherites. Reinisch, in 1884, obtained magnificent results with double salts of ammonium and sodium, prepared by dissolving $1 \mathrm{~g}$. of ammonium sulphate and $2 \mathrm{~g}$. of crystallised sodium sulphate in 80 parts of water and adding 10 per cent. of nitric acid. With a magnification of 20 the spheres were $2 \mathrm{~cm}$. in diameter. They consisted of needles arranged radially with a notched edge, showed a grey shadow cross and three wide concentric colour rings, but only as long as the crystals were still moist. After drying the crystals appeared silvery white. Very beautiful results were obtained with erbium yttrium sulphate without the addition of nitric acid.

Numberless examples are known of organic compounds which crystallise as spherites. These are usually substances of high mọlecular weight, or such as are probably much aggregated in solution. Inulin is deposited from water as spherites only when a saturated solution is slowly crystallised. Quick evaporation forms amorphous crusts. Evaporation of a drop of an aqueous solution on glass leaves a transparent horny fibrous mass. If the evaporation is slowed, as by placing a coverglass with a hanging drop of the inulin solution upon a moist cardboard frame, good doubly refracting spherites are obtained 
[Leitgeb, 1888; Fischer, 1902]. The growth of spherites of amylodextrin was observed by Syniewski [1902]. "If a drop of the [formaldehyde] solution is placed on a microscope slide, covered with a cover glass, and brought under the microscope, after an hour the commencement of the crystallisation is seen. Needle-like crystals shoot at first to grouped star-like bundles. After a time these bundles become composed of regular concentrically arranged layers of extraordinarily fine needles directed towards the centre, which then show a thousand structures like starch grains. This imitation of starch grains goes so far that aggregated grains are also seen to originate. All these grains are composed of doubly refracting needles and show beautifully, in polarised light, the well known dark cross of the starch grains." The same author has shown that starch grains consist wholly of a single substance and that the amylocellulose, of which the outer skins of the grains were supposed to be formed, is a 'decomposition product. Having regard to Bütschli's [1898] preparation of artificial starch grains by crystallisation of starch in presence of gelatin, little doubt remains that the starch grains are themselves identical in origin and structure with spherites.

The production of large crystals by slow diffusion, as through strips of filter paper [Monnier, 1874], or gels [Vesque, 1874], is well known. In 1879 Ord observed the precipitation of calcium oxalate by diffusion of ammonium oxalate through an isinglass gel in an open tube immersed in calcium chloride solution. The precipitate consisted first of growth forms changing into spherites towards the calcium chloride, which must have been bypotonic. The very frequent occurrence of spherites in gels was pointed out by Hatschek and is being studied by the writer. Spherites of various difficultly soluble inorganic salts have been prepared by the diffusion of reagents into gels containing solutes with which they react. Frequently the precipitate formed in the upper regions of the gel, where the reagents are more concentrated, is composed of small needles or tiny regular crystals. As the dilution of the reagents increases and the velocity of crystallisation diminishes, the particles become much aggregated and approach spherical shape, often becoming perfect spheres in the lower nearly exhausted regions of the gel. Among others, good examples of spherites of silver bichromate, copper and calcium carbonates have been obtained. The former are opaque, the two latter substances show black crosses in polarised light, and the larger spheres colour rings. Spherites of manganese polysulphide have been produced over $1 \mathrm{~cm}$. in diameter. These are opaque, but have the same banded structure. They resemble closely the large spheres, several inches in diameter, of magnesian 
limestone occurring in the Permian beds at Fulwell Hill, Sunderland, described by Abbott. These results show that the formation of spherites is not induced by quick crystallisation. In all the cases quoted it is, however, reasonable to assume a state of molecular aggregation in solution. The slow diffusion in the presence of protective colloid in gels favours the formation of such molecular aggregates in solution. Supersaturation, especially in viscous media and with bodies of high molecular weight, has the same effect, and since the crystallisation of a substance usually begins from a supersaturated solution, the first appearance of spherical masses would be in accordance with an existing state of aggregation. Consequently it may be doubted whether the "droplets" frequently recorded are invariably liquid. If they were so, the cause of their further development into spherites; instead of crystals, in some cases, would still require explanation. There are, however, many bodies of low melting point which undoubtedly appear at first in the form of liquid drops. From Hardy's [1900] experiments with gelatin sols treated with alcohol, this may possibly be the case in the gelation of gelatin. In accordance with Masson's [1891] theory of solution, gelatin may melt in water at a lower temperature than that of complete miscibility, below which there would exist two phases, one of melted solid containing a little water, and the other of water saturated with gelatin. The velocity of crystallisation of gelatin is so slow that even the melted solid phase may form spherites. In any case aggregation in solution appears to be a principal cause of the formation of spherites. Another important factor must be the viscosity of the liquid medium, which restricts the movement of the unwieldy molecules or molecular aggregates. Under the influence of the feebler forces of crystallisation, consequent upon the size of the particles, these have the less opportunity to pack themselves into regular crystals.

From the rapidity of the formation of the layers, Leitgeb [1888] has shown that they cannot be influenced by outside causes, but must be due to changes in the concentration of the solution in the immediate neighbourhood of the crystals. Observation of banded precipitates formed by diffusion of reagents into gels, indicates that the zonal structure is due to adsorption, by the precipitate, of solute from the gel, until the region next the precipitate is exhausted of solute, and the reagent is able to diffuse further into the gel before forming a fresh band [Bradford, 1916, 1, 2]. A similar explanation may be given of the zonal structure of spherites. Since these consist of numerous ultramicroscopic individual particles, their specific surface will be very large. Consequently the solute will be rapidly adsorbed and the 
concentration in the region next the spherite reduced. If the concentration fall sufficiently, crystallisation will be monentarily suspended or its velocity reduced until fresh supplies of nutrient material arrive.

These considerations lead to the conclusion that substances of high molecular weight and such as exist in solution in the aggregated form, tend to crystallise in the form of spherites. It is reasonable to suppose, therefore, that the granules seen ultramicroscopically in gels, which must have great specific surface, actually consist of these spherical aggregates.

Spherites of gelatin can easily be produced of microscopic size. A sample of dialysed gelatin precipitated by alcohol, was dissolved in about 100 parts of water and poured slowly into alcohol. The smallest trace of the precipitate brought on to a microscopic slide, showed a mass of innumerable transparent granules, round the edge of which individual grains could be seen. Under a magnification of about 800 the granules appeared perfectly spherical and translucent of about 0.5 to $2 \mathrm{~mm}$. diameter (Fig. 1). No connection between the grains could be seen.

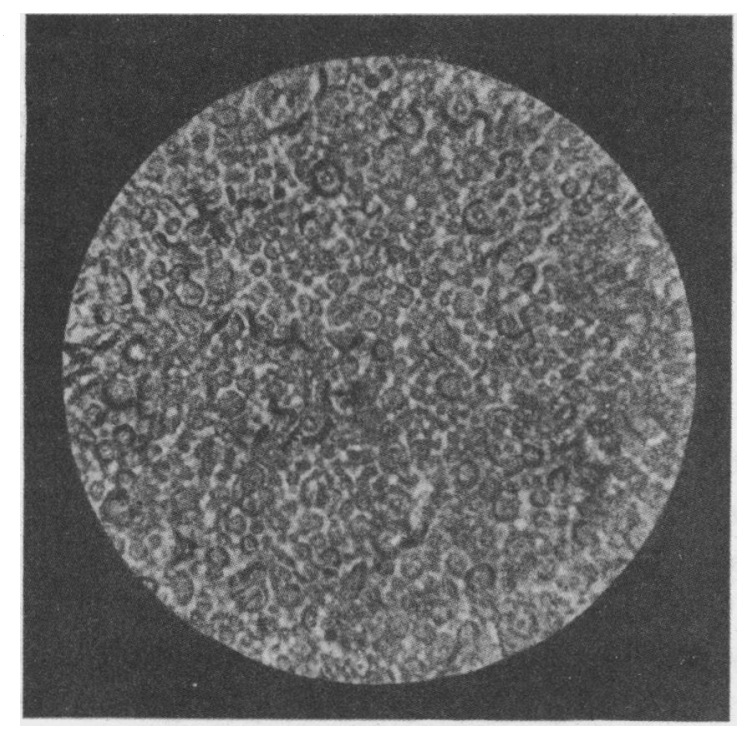

Fig. 1. Gelatin precipitated by alcohol $\times 800$.

The question next arises as to whether the micellae are themselves aggregated to form structures within the gel. On account of the small size of the micellae, the problem is the more difficult to solve. Ultramicroscopic examination of untreated gels gives no indication of such aggregation. 
Moore [1915] observed chains of globules in silicic acid sols. The possibility of the existence of microscopic filaments has been suggested [Bradford, $1917,2]$. This is not supported by the viscous properties of gels which permit the movement of solid particles and gas bubbles within them. Aggregation of the micellae is possibly more likely to occur in the surface skins of gels owing. to the influence of adsorption and evaporation in increasing the concentration. Hitherto the influence of these skins on the properties of gels does not seem to have received sufficient attention. Moreover, if one region of a sol should gelate before another, adsorption might cause a rhythmic variation in the density of distribution of the gel particles similarly to the bands in spherites. The possibility of aggregation of the micellae within the gel has been investigated ultramicroscopically by Moeller [1916, 1917], who considers that the micellae are united into fibrils, like those in organic membranes, but which are obscured by the thickness of the layers viewed ultramicroscopically. To overcome this difficulty he observes very thin layers of gelating sol upon a microscope slide with a cardioid ultracondenser, and, before gelation, he adds alcohol or some tanning material. . In other cases the surface of the gel is treated with the tanning solution, and the appearance observed before diffusion has reached the interior of the gel. These methods are not very different from those employed by Bütschli and others for microscopic examination. Unfortunately both methods are open to great objection. In the first case the addition of precipitating reagents to the sol fundamentally transforms the character of the crystallisation process, and it may be observed that the structures seen were immediately broken up by slightly disturbing the slide during gelation. In the second case, as has been shown, the properties of the surfaces of gels are likely to differ from those of the interior. In both cases the formation of precipitates by the reaction of the tanning reagents, such as chrome alum, with the impurities of the nature of calcium salts in the gel does not appear to be excluded. The presence of such impurities in the gelatin used is confirmed by a photograph showing spherites, produced in the drying gel, exactly resembling the form in which calcium sulphate occurs in gelatin. Such precipitates would undoubtedly be produced in any but the most carefully purified gelatin and might show structures similar to those observed. Moreover, it seems probable, as Wilson has pointed out, that gelatin forms insoluble compounds with alum and chrome tanning material. These would tend to be precipitated in the banded form. The production of similar laminated or striated forms by the crystallisation of salts in gelatin films, is well known, and originates from the action of adsorption and 
diffusion as in the Liesegang phenomenon [Bradford, 1916, 1, 2]. From the similarity of the structures seen with Liesegang's rings, Moeller argues, in the second.paper, that the latter are due to the pre-existence of laminated structures in the gel. This is certainly controverted by many. facts : such as that the distance apart and the width of the precipitated bands can be determined, in a given gel, by a suitable choice of the concentrations of the reacting solutions; that the orientation of the bands is always at right angles to the diffusion path; and that similar banding can be produced in sand columns and filter paper. Moeller also obtains various shrinkage figures, like those previously studied by Liesegang and others, in the surface of gelatin gels by the action of water vapour, hot water, or drying. From these experiments Moeller concludes that, of the $\alpha$ - and $\beta$-gelatin of which the gels are composed, the $\alpha$-gelatin forms fibrils which intertwine to form a network, in the meshes of which is enclosed the $\beta$-gelatin, or gelatose, as a structureless mass in solution. The theory is then extended to include all gels. While however the possible existence of fibrils in the interior of gels cannot be definitely excluded at present, it may be considered that the available evidence is insufficient to establish their presence.

\section{The structure aNd properties of Gels.}

From the point of view of the foregoing, the natural emulsoid gels may be regarded as two phase systems comprising innumerable ultramicroscopic globular crystalline molecular aggregates, or probably spherites, in, and around, which the mother liquor, from which the disperse phase has crystallised, is held by molecular and capillary forces.

Owing to the enormous specific surface, the state of a portion of the liquid phase will differ from that of a free liquid. Assuming with Laplace that the surface tension and intrinsic pressure of liquids may be attributed to the molecular attractive forces, the molecules in the outer skin of liquid, at a liquid-air surface, experience a force directed normally towards the surface which is due to the unbalanced molecular attractions, and, in this surface layer, the intrinsic pressure of the liquid falls rapidly towards that of the atmosphere. Conversely, if it be allowed that solid molecules have usually a greater attraction for liquid molecules than these for themselves, of which there are many indications, it may be supposed that, in a liquid-solid surface skin, the internal pressure tends rapidly to approach the intrinsic pressure of the solid, and may be many times that of the free liquid. The dispersion medium in 
gels will exist therefore in at least two states, (1) the adsorbed liquid, i.e. that in the skins surrounding the solid phase which will be under an enormously increased pressure and (2) the liquid in the larger interstices of the gel held by capillary forces. Moreover, owing to the action of adsorption and, especially, of evaporation, the boundary surface of gels will be composed of a more or less tough skin which may materially affect the apparent properties of the gel. This skin is much more noticeable in some cases, and varies -with the age and previous environment of the gel. Thus gelatin gels have very tough 'skins, agar gels, much less so, and silicic acid gels hardly noticeable skins. The cohesion of gelatin, agar and silicic acid gels varies in the same way, and since, on cutting the surface of a gelatin gel, the cohesion of the gel is enormously reduced, it seems probable that the cohesion resides largely in the surface skin. Determination of the breaking stress of 15 per cent. gelatin and 2.5 per cent. agar gels gave the small value of about $105 \mathrm{~g}$. per sq. cm. for the latter, as the mean of three experiments, while the gelatin gel did not break with a load of $1282 \mathrm{~g}$. per sq. $\mathrm{cm}$. All these gels were about 30 hours old. It is evident therefore, that; in considering the stress-elongation curve of gels, the elasticity of the surface skin must be taken into account, and the elastic properties of this skin deserve further study.

The vapour pressures of gelatin-water mixtures have recently been studied by Gerike [1915]. Gelatin gels show a slight lowering of the vapour pressure as compared with pure water, and the concentration of the gel is of little importance below 70 per cent. gelatin. From about this composition the lowering of vapour pressure is proportional to the increase in percentage composition of gelatin; at about 94 per cent. there is a second inflexion in the vapour pressure curve. Gerike also found that the nature of the surface has a considerable influence on the behaviour of the gel in an atmosphere of water vapour. These results appear to indicate the existence of the gel water in three states, (1) free water containing solated gelatin, (2) water contained in fine capillaries, and (3) a small mass of more firmly bound water, which may correspond to that in the surface skins around the solid phase and may be called the adsorbed water.

Zsigmondy [1911] has shown that the von Nägeli bypothesis supplies the explanation of the curious hysteresis cycle in van Bemmelen's [1878, 1880] well known vapour pressure isotherms for silicic acid gels. Silicic acid gels dry to a clear transparent finely porous gel, which is practically constant-in volume and only permits of volume changes within its elastic limits. The hysteresis cycle corresponds to this constant volume gel. 
During the progressive dehydration along the first descending branch of the curve, the gel is decreasing in volume, and the gel capillaries develop menisci at the gel surface, concave to the vapour phase, with increasing curvature corresponding to the fall in the vapour pressure. At the commencement of the hysteresis cycle the radius of curvature of the liquid menisci has reached its lowest value. The radius of curvature has now become approximately equal to the radius of the capillary. Such a meniscus has a maximum pull on the layer of liquid below, in consequence of which the gel walls are drawn together and the gel skeleton as a whole undergoes compression. The tension causes rupture of the liquid in the interior of the gel, and the appearance of the gas phase. The occurrence of innumerable gas bubbles causes the opaqueness which begins at this point. The bubbles are larger than the size of the interstices between the micellae, and envelop a number of particles of the solid phase. Finally, when the emptying of the capillaries is complete, at the lower point of the cycle, the bubbles have all coalesced and the opaqueness has disappeared. These facts indicate an extraordinarily fine structure. Larger discontinuities, of the order of Bütschli's honeycombs, must cause permanent opaqueness.

The ascent of the steeper branch during reimbibition is due to the fact that the unwetted walls develop liquid menisci with greater radii, and consequently higher vapour pressure, than before. The greater water uptake during the last part of this branch corresponds to the enlargement of the capillary volume in consequence of the diminished pressure in the gel occasioned by the gradual flattening of the menisci.

For such a gel the size of the capillaries can be calculated, and, for a reduction of vapour pressure of $6 \mathrm{~mm}$., Zsigmondy finds a capillary radius of about $2 \cdot 5 \mu \mu$.

This was completely confirmed by Anderson [1914], who replaced the imbibition water by alcohol and benzene, and determined the vapour pressure isotherms in a vacuum apparatus. The curves were of exactly the same

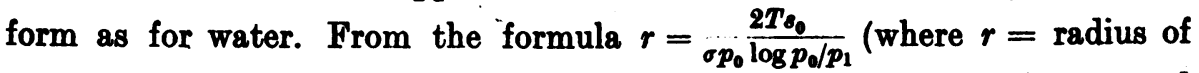
capillary, $T=$ surface tension of imbibed liquid, $s_{0}=$ density of saturated vapour, $\sigma=$ density of imbibed liquid, $p_{0}=$ ordinary vapour pressure of imbibed liquid, and $p_{1}=$ vapour pressure of liquid in capillaries) Anderson found the values:

$\begin{array}{ccc}\text { Imbibed liquid } & p_{1} \text { in } \mathrm{cm} . \mathrm{Hg} . & r \text { in } \mu \mu \\ \text { Water } & \mathbf{0 . 8 4} & 2.74 \\ \text { Aloohol } & 2 \cdot 12 & 5.58 \\ \text { Benzene } & 2.9 & 2.99\end{array}$


These figures indicate that the diameter of the ultimate particles composing the gel micellae is about $5 \mu \mu$. Taking the thickness of the surface skin of adsorbed liquid, as calculated from van der Waal's equation, at about $0 \cdot 15 \mu \mu$, and assuming a specific surface corresponding to spherical particles $5 \mu \mu$ in diumeter, the total volume of adsorbed liquid in a so-called $10^{\circ}$ per cent. gelatin gel, containing 8.5 per cent. of dry gelatin, would be about 0.8 per cent. This figure agrees fairly with the value calculated from Rodewald's data below.

Organic hydrogels such as gelatin differ from silicic acid gels in the method of their formation. Whereas the gelation of the latter is irreversible, the gelation of the organic gels is reversible. The cause of this difference lies in the solubility of the organic gel substances in the dispersion medium, as has already been shown. This solubility affects the behaviour of the gel on drying. Evaporation leads to gradual deposition of solid in the pores of the micellae as these are pulled together by the increasing curvature of the liquid menisci, with the result that the gel dries to a transparent hard mass. The rehydration process must include the resolation of the deposited excess of gel substance before the full amount of water can be taken up. This is the reason why the organic gel substances will not absorb liquids in which they are insoluble. Bütschli showed, however, how to substitute such liquids for the water in a swollen gel, and Bachmann [1917] utilised his method to obtain constant volume gels of which the vapour pressure isotherms might be studied. The procedure consists in gradually hardening the gelatin, and, at the same time substituting alcohol for the water, by immersing the hydrogel in aqueous alcohol of gradually increasing concentration. In this way a hardened gelatin alcohol-gel is obtained in which the alcohol occupies the same volume as did the water; and the alcohol can be changed for benzene, xylene and other liquids. The vapour pressure isotherms of these hardened gels were of the same type as the van Bemmelen curves for silicic acid, showing a similar hysteresis cycle corresponding to an opaque appearance in the gel. Bachmann also studied a number of other porous substances, such as hydrophane, permutite and wood charcoal, and concluded that there is a series of chemically very different substances with finely porous gel structure, showing similar vapour pressure isotherms, which correspond to the reduced vapour pressure of a liquid inclosed in amicroscopic capillaries. All the curves include a hysteresis cycle analogous to those discovered by van Bemmelen for the gels of copper oxide, iron oxide and silicic acid. The type of the hysteresis cycle is dependent neither on the nature of the imbibed liquid nor 
on that of the gel skeleton. Bachmann calculated that the capillaries are 30-300 times smaller than those required by Bütschli's honeycomb structure, which would have the enormous size of $700-800 \mu \mu$, and could play no part in the reduction of the vapour pressure.

From experiments on the imbibition of pure chemical compounds, Katz [1917] found that the degree of swelling is independent of the chemical nature of the swelling substance. The curves showing the relation between the degree of swelling and the vapour pressure, volume change, and heat of swelling, are similar to the corresponding curves for mixtures of water with non-volatile liquids such as sulphuric acid and glycerol. From this Katz concluded that the swelling process is to be regarded as the formation of a solid solution, and is incompatible with von Nägeli's theory. On the contrary, the question may be asked, whether the causes underlying the phenomena in both cases are not of the same nature, and whether it may not be hoped that the explanation of the similarity will be found in the sphere of action of the intra-molecular forces. This view of the imbibition process is in conformity with Proctor's experiments [1914] which show that the phenomena are dependent on the relation between the osmotic pressures and equality of products of the hydrolysable gelatin salt which is formed and of the external acid solution.

Some indication of the enormous pressures experienced by the adsorbed liquid in gels is afforded by Schroeder's experiments with gelatin [1903] and by Reinke's work with the oedometer [1879]. Working with discs of Laminaria, the latter showed that gels, separated from water by an osmotic membrane, develop pressures, in equilibrium with the water content of the gel, increasing to 41 atmospheres as the water content falls to 16 per cent. of the air-dry gel. Since air-dry Laminaria contains about 20 per cent. of water, the pressures under which the last traces of water are adsorbed must be enormous. It is impossible to ignore the apparent analogy between these pressures and the osmotic pressures of solutions, with the suggestion that both originate in the molecular attractions of the liquid and solid phases or of solvent and solute. Indeed the study of colloids abounds with indications that the difference between true and colloid solutions, and again between these and gels, is merely one of degree, and that the same general laws are applicable to all. In this connection it will be remembered that the close relation between the properties of sols and gels was pointed out by Arisz [1915] who concluded that gels should be regarded as liquids of great viscosity.

Reinke's experiment does not lead to a direct estimation of the pressure 
of the adsorbed liquid in gels. More insight is obtained by examination of the phenomena of swelling.

The total contraction of gel and water on imbibition by gelatin has been studied by Lüdeking [1888]. Unfortunately he used air-dry gelatin, which still contains about 15 per cent. of water, so that the volume changes observed were only a fraction of those to be expected with a dry gel. The density of the air-dry gelatin was 1-412. From the densities found by Luideking, the contractions in cc. have been calculated assuming the water alone to have decreased in volume.

$\begin{array}{lccc} & \begin{array}{c}\text { Specific } \\ \text { volume }\end{array} & \begin{array}{c}\text { Volume in cc. } \\ \text { of gelatin in } \\ 100 \text { g. of gel }\end{array} & \begin{array}{c}\text { Contraction per } \\ \text { cc. of water }\end{array} \\ \text { Air dry gelatin } & \mathbf{0 . 7 0 8 2} & - & - \\ 10 \text { g. gelatin }+90 \text { g. } \mathrm{H}_{2} \mathrm{O} & \mathbf{0 . 9 3 5 4} & \mathbf{7 \cdot 0 8} & \mathbf{0 . 0 4 0} \\ \mathbf{2 5} \text { g. gelatin }+75 \text { g. } \mathbf{H}_{2} \mathrm{O} & \mathbf{0 . 8 8 1 0} & 17 \cdot 70 & 0.063 \\ 50 \text { g. gelatin }+50 \text { g. } \mathrm{H}_{2} \mathrm{O} & -0.8052 & 35 \cdot 41 & 0.098\end{array}$

These small contractions indicate that the first portions of water taken up are the more compressed. The maximum contraction corresponds to a density for the adsorbed water of about $1 \cdot 11$.

Information of more value is given by Rodewald's investigations on the contraction in total volume of starch and water during swelling [1897]. Rodewald used starch dried over sulphuric acid. This still contains some water, since drying in vacuo at $100^{\circ}$ is necessary to drive off all the water [Dafert, 1887], but the contractions obtained should be much greater than with an air-dry gel. Assuming that the contraction is confined to the water, the volumes of $1 \mathrm{~g}$. of water at each concentration have been calculated from Rodewald's results.

\begin{tabular}{|c|c|c|c|c|}
\hline $\begin{array}{l}100 \mathrm{~g} \text {. starch } \\
\text { dried over } \mathrm{H}_{2} \mathrm{SO}_{4} \text {, } \\
\text { containing water }\end{array}$ & $\begin{array}{l}\text { Specific } \\
\text { Volume }\end{array}$ & $\begin{array}{c}\text { Vol. of } \\
\text { adsorbed } \\
\text { water in cc. }\end{array}$ & $\begin{array}{l}\text { Vol. of } \\
1 \text { g. water } \\
\text { in cc. }\end{array}$ & $\begin{array}{c}\text { Vol. of } 1 \mathrm{~g} \text {. additional } \\
\text { water, after subtracting } \\
\text { vol: }(=3.01 \mathrm{cc} \text {. }) \text { of first } \\
9.8 \mathrm{~g} .\end{array}$ \\
\hline $0^{-}$ & $0 \cdot 72585$ & - & - & - \\
\hline $1 \cdot 17$ & 0.72124 & 0.38 & 0.33 & - \\
\hline 2.69 & 0.71319 & 0.78 & 0.29 & - \\
\hline 5.02 & 0.70486 & 1.54 & 0.31 & - \\
\hline $7 \cdot 40$ & $0 \cdot 69881$ & $2 \cdot 67$ & $0 \cdot 36$ & 一 \\
\hline $9 \cdot 70$ & $0 \cdot 69344$ & 2.79 & $\mathbf{0 . 2 9}$ & - \\
\hline 13.41 & 0.68727 & 6.60 & 0.49 & 0.91 \\
\hline 14.95 & $0 \cdot 68555$ & $8 \cdot 22$ & 0.55 & 1.01 \\
\hline $19 \cdot 24$ & 0.68111 & $9 \cdot 51$ & 0.49 & 0.68 \\
\hline
\end{tabular}

It will be noted that up to about 9.8 per cent. of water, in addition to that already contained in the starch, the contraction of the adsorbed water is practically constant. Subtracting the volume of this 9.8 per cent. of water from the calculated volumes of adsorbed water at larger concentrations gives 
practically no contraction for the additional water, except in the last case. From these figures it is possible to get a very rough idea of the pressures under which the water is imbibed.

The work of Worthington, Richards and Matthews, and others indicates that the product of the internal pressure, $P$, of a liquid and its compressibility, $\beta$, is approximately constant, i.e. $P \beta=C$. Amagat's figures [1893] show that this is not quite true for water. But, from the anomalous nature of water as a liquid, it is probable that the relation between its internal pressure and compressibility is not quite simple. Since the available data are so meagre and have not been derived from the study of dry gels, all that can be expected is to show that the theoretical considerations lead to values of the right order.

Assuming the relation $P \beta=C$, and taking $P_{0}=1.1 \times 10^{4}$, and

$$
\beta_{0}=5 \cdot 2 \times 10^{-5} \text {, gives } C=0.572 \text {. }
$$

And since

$$
\begin{gathered}
\beta=-\frac{1}{V} \frac{d V}{d P} \\
\therefore \frac{d V}{V}=-0.572 \frac{d P}{P} \\
\text { or } \log _{10} \frac{V_{0}}{V^{1}}=0.572 \log _{10} \frac{P_{1}}{P_{0}} .
\end{gathered}
$$

Since the first $9 \cdot 8 \mathrm{~g}$. of water become $3.01 \mathrm{cc}$., the average pressure producing this contraction is given by

$$
\log \frac{9 \cdot 8}{3 \cdot 01}=0.572 \log \frac{P_{1}}{1 \cdot 1 \times 10^{4}}
$$

which gives $P_{1}=8.6 \times 10^{4}$.

From the last line in the table, the additional $9.44 \mathrm{~g}$. of water adsorbed become 6.50 cc., from which $P_{2}=2 \cdot 1 \times 10^{4}$.

The heat evolved in the contractions should be given approximately by

$$
\begin{gathered}
Q=\frac{\left(P_{1}-P_{0}\right)\left(V_{0}-V_{1}\right) \times 10^{4}}{42 \times 10^{8}} \mathrm{cal} . \\
\therefore Q_{1}=1.2 \times 10^{4} \mathrm{cal} \text {, and } Q_{2}=7 \times 10^{2} \mathrm{cal}
\end{gathered}
$$

which is negligible as compared with $Q_{1}$. This is the heat evolved by $100 \mathrm{~g}$. of starch. The heat evolved per gram is therefore $120 \mathrm{cal}$.

Rodewald found about $30 \mathrm{cal}$. per $\mathrm{g}$. for starch, which is only a quarter of this amount. There is, however, another change which takes place during swelling, in addition to the condensation of the water, and this causes absorption of heat. In accordance with Laplace's conception, the presence of a liquid will reduce the unbalanced attractions in the surface layer of the solid, in consequence of the attraction of the liquid molecules in the opposite sense. The surface tension of the solid will therefore be decreased, and 
expansion of the surface layers will take place, with absorption of heat. The observed heat of imbibition is, therefore, less by this amount.

The heat of imbibition of gelatin was determined by Wiedemann and 'Lüdeking [1885]. Here again unfortunately air-dry substance was used, so that a value of only 5.7 cal. per g. was obtained. Before the employment of only air-dry substance was realised, the low value of the heat of swelling seemed a serious difficulty in working out the present theory. A determination of the heat of swelling of gelatin dried at $110^{\circ}$ gave $32.96 \mathrm{cal}$. per g. as the mean of two experiments, or, allowing for the heat, absorbed by the solation of gelatin by the water in the calorimeter, according to Wiedemann and Lüdeking's figures, the heat of swelling becomes $33.25 \mathrm{cal}$. This value is very similar to that obtained for starch.

The so-called "thermal anomaly" of gels was discovered by von Bjerkén [1891]. Arguing from a supposititious analogy with rubber, von Bjerken expected to find that stretched gels would likewise contract on heating. From a number of experiments which were confined to gelatin gels, of which neither the quality, composition, nor form is recorded, the expectation appeared to be substantiated. No explanation of such a phenomenon is afforded by the theory considered in this paper. However, having regard to the tough skin which forms on the surface of gelatin gels, it seemed probable that such gels would contract in one direction when heated, owing to an approach to spherical shape under the influence of thermal expansion. This contraction would be dependent on the shape of the gel, and independent of any stretching force applied to the gel except indirectly as the result of a change induced in its shape. The contraction would also be affected by the variation in the elasticity of the outer skin with age or change of environment. The single case of a non-stretched gel recorded by von Bjerken gave a very slight expansion in the unstretched state, and only a small contraction when stretched. The definite expansion is curious, since experiments show that expansion in the direction of greatest length is due to relaxation of the skin and proceeds without limit until the gel melts. This suggests that, in the unstretched state, the gel in question had its greatest diménsion in some other direction than the one under observation.

Von Bjerkén himself records a noticeable small gradual expansion, which was superposed upon the contraction effect. Doubtless this was due to the gradual relaxation of the surface skin. It was therefore determined to reinvestigate the thermal behaviour of gelatin ribbons and cylinders, and the results of over seventy experiments completely fulfil expectations. The 
gels showed either unlimited expansion, or contraction which varied with the age or previous history of the surface skin. Cutting or injuring the skin caused expansion instead of contraction.

No exact formula can be obtained to express the relation of the contraction to the form of the gel. An approximate one may be arrived at by supposing the volume, $V=\pi r^{2} h$, of a cylinder of gel to increase, while the surface, $S=2 \pi r h+2 \pi r^{2}$, remains constant ${ }^{1}$. This gives

or

$$
\begin{gathered}
d S=2 \pi h d r+2 \pi r d h+4 \pi r d r=0 \\
d r=-\frac{r d h}{h+r} .
\end{gathered}
$$

The change in volume,

$$
\begin{aligned}
d V & =2 \pi r h d r+\pi r^{2} d h \\
& =\pi r^{2} d h-\frac{2 \pi r^{2} h d h}{h+r} \\
& =-\frac{\pi r^{2}(h-r)}{h+r} d h \\
\therefore & \frac{1}{V} \cdot \frac{d V}{d \theta}=-\frac{1}{h} \cdot \frac{h-r}{h+r} \cdot \frac{d h}{d \theta} .
\end{aligned}
$$

A cylinder is, however, not so convenient to work with as a ribbon, since there is danger that thermal equilibrium may not be reached during the rapid temperature changes.required to prevent the gel melting. Only a still more approximate formula for a ribbon can be deduced. By writing $\rho=\frac{\sqrt{a+b}}{2}$, where $a$ and $b$ are the shorter dimensions of the ribbon, the equation becomes

$$
\frac{1}{V} \cdot \frac{d V}{d \theta}=-\frac{1}{h} \cdot \frac{h-\rho}{h+\rho} \cdot \frac{d h}{d \theta} \text {. }
$$

Since the surface skin of agar gels is very much softer than that of gelatin gels, it would be expected that the former would show no contraction on heating. This was found to be the case. It may therefore be regarded as established that the contraction in greatest length of gelatin gels when heated is due to the constricting effect of the surface skin as the gel expands in volume in accordance with its coefficient of thermal dilation, and is independent of any stretching force applied to the gel.

In so far as the phenomena exhibited by gels depend on the interrelation between the liquid and solid phases, they should be influenced by the well known lyotrope series of dissolved salts, which affect the surface tension and the affinity of the liquid and solid phases. The connection between adsorption of solvent and swelling, and the similar effect of the Hofmann series of anions on both was pointed out by Lillie [1907]. He suggested that osmotic pressure is due to adsorption of solvent by the dissolved particles, and showed

1 Actually if the surface were inelastic the gel could not change in shape. 
that the osmotic pressure of emulsoid sols is decreased by salts in the same order, $\mathrm{CNS}<\mathrm{I}<\mathrm{NO}_{3}<\mathrm{Br}<\mathrm{Cl}<\mathrm{SO}_{4}<\mathrm{CO}_{3}$ as in the case of solutions. The diminished pressure would not appear to be due to increased aggregation of the sol particles, since the precipitating effect of the cations has not commenced at the concentrations employed, although anions hinder the precipitation in the opposite order [Pauli, 1913]. The same relations were found by Hatschek for the clearing of oil emulsions. The temperature of gelation should however be depressed in the order $\mathrm{CNS}>\mathrm{I}>\ldots>\mathrm{CO}_{3}$ as was found by Levites [1908]. The change in the viscosity with time [Schröder, 1903] may be deduced in the same way as due to a diminution of the precipitating forces and the formation of fewer crystallisation centres, or sol particles. If the velocity of crystallisation is sufficiently reduced, gelation may be altogether prevented, as by the addition of the thiocyanate anion. The solubility of the colloid particles is temporarily increased thereby, their aggregation hindered and the sol stage prolonged. In a similar way the elasticity of gels is increased by salts which favour imbibition and vice versa. The double refraction of deformed sols shows the same lyotrope effect [Lieck, 1904].

\section{Experimental.}

The gelatin used in all, except the earliest, of these experiments was Nelson's "Crystal" leaf gelatin. It contained 15.41 per cent. of moisture, 1.66 per cent. of ash and only a trace of phosphate. The ash appeared to be due mostly to the presence of calcium bicarbonate in the gelatin.

A quantity of this gelatin was soaked for 6 days in running distilled water and quickly evaporated until the gel corresponded to 10 per cent. of the original gelatin. This gel gave no precipitate when treated with limewater, or sodium phosphate solution, and only a trace of precipitate in the form of finely divided bands, with silver nitrate solution. It did not give the Liesegang phenomenon until citric acid had been added. Contrary to Liesegang's experience it was then unnecessary to add additional gelatose.

The gel so prepared was, however, quite unsuitable for the investigation of the thermal anomaly of gelatin, since the gelatin had almost completely lost its coherence, together with its property of forming a hard surface skin. It was impossible to mould a cylinder or ribbon which could be handled without breaking. This behaviour was identical with that of a sample of gel similarly prepared from another specimen of gelatin. The gelatin was further purified by two precipitations by pouring the aqueous sol into alcohol. 
A number of atbbons made from this gelatin had also very soft surface skins and were equally fragile. From this it appears that the cohesion of gelatin is due to a water soluble substance, probably gelatose, which is removed by soaking. Consequently the leaf gelatin was used for the experiments in its original state. The agar gels were prepared directly from ordinary commercial shredded agar.

The so-called "Thermal anomaly" of gels.

The apparatus employed by $v$. Bjerkén for the observation of this property consisted of an apparently thick glass beaker, provided with a stirrer, and containing paraffin oil in which the ribbon of gel was mounted. The beaker was surrounded with an outer jar containing water, by changing which the temperature of the paraffin oil bath was regulated. Since the gelatin is heated to about $40^{\circ}$, which is much over its melting point, it is important that the temperature changes should be as rapid as possible. Consequently a modified form of apparatus was used. This comprised a thin glass tube $10 \times 2 \mathrm{~cm}$., with an overflow lip above, and having a side tube $1 \mathrm{~cm}$. diam. at the base, where the main tube was constricted to $5 \mathrm{~mm}$. diam. Through the constricted tube a glass rod passes freely, provided with a platinum hook above for the attachment of the gelatin ribbon. The space between the rod and the tube was closed by an outer sleeve of rubber tube. The rod was long enough for the hook to be passed to the top of the main tube when fitting the ribbon. It was then carefully drawn down, until the gel was in position, and securely clamped in place. By means of the side tube a rapid stream of liquid could be passed through the main tube from either of two thermostats at the temperatures selected for the experiment. These were usually that of the room and about $40^{\circ}$. The leading tubes were $1 \mathrm{~cm}$. in diameter and, to avoid constriction, rubber connections and pinch cocks were employed. The side tube was fitted at right angles below with a waste tube, of the same diameter, for the rapid emptying of the main tube. The fluid found most convenient was liquid paraffin B.P. Observation showed that the change in length of the gel when immersed in water is inappreciable during the short time of the experiment, and this liquid was used on some occasions as a check. Water has also the advantages of greater heat capacity and mobility. When using liquid paraffin a small correction for the difference in temperature of the bath from that of the thermostat must be applied. With water there is no correction.

The gel cylinders or ribbons were moulded with metal plates embedded in the surfaces at the two ends. The plates were provided with holes through 
which wires could be hooked for attachment. The lower end of the gel was hooked to the sliding glass rod, and the upper end was connected to a light lever and pointer, by means of which the position of the end of the gel could be estimated, with the aid of a lens, to within $0.001 \mathrm{~mm}$. The weight of this lever caused a pull of only $6.5 \mathrm{~g}$.

The preparation of the cylinders and ribbons was a matter of considerable difficulty, since it was essential to secure gels of which the surface was uninjured. Glass moulds, which were afterwards broken, were of no use. Moulds of silver foil formed round a glass tube and clamped down the side by a bull-dog clip, the edges of which had been filed square, seemed most convenient. The lower end of the tube so formed was closed by a split cork carrying the tongue of the circular plate carried above for the attachment of the gel, and was luted with paraffin wax if necessary. The upper plate was suspended by a pin through the perforation of the tongue, the cylinder filled with gelatin and allowed to set. The clamp was then removed and the cylinder weighed and slipped into a small test tube which was corked until required for use. The ribbons were moulded in troughs formed of extra stout tin foil, and kept in corked test tubes. The rate of loss of moisture from the gel was found to be fairly constant at about 0.6 per cent. in the time, about 20 hours, before measuring the expansion. From the time of taking out of the tubes it. was impossible to control the loss of water. The compositions of the gels are therefore given as they were prepared, and their history recorded in èch case. As a guide to their water content, the rate of loss of moisture by a sugar loaf of 10 per cent. air-dry gelatin weighing $15 \cdot 147 \mathrm{~g}$. exposed to the air under a raised inverted funnel was determined, see Fig. 2. To open the mould, the sheet metal was carefully peeled off the gel. Usually the surface of the gel was little damaged in the process, but it was never obtained quite perfect. Accordingly the gel was hung on a hook and painted all over with a hot gelatin sol of the same concentration. After drying for several hours in the air, the gel was carefully mounted in the apparatus and the cold liquid run through for about 30 seconds to insure temperature equilibrium. If the surface of the gel was even slightly injured in mounting, the gel subsequently gave no contraction. After 30 seconds the scale reading was taken, the cold liquid quickly run out, and a rapid current of hot liquid maintained until the maximum contraction had been recorded. This took about 15 seconds in liquid paraffin and half that time in water. The current of hot liquid was immediately changed for cold, before the gel melted, when the gel invariably expanded to beyond its original volume, the expansion continuing for some 
time. This appeared to be due to the softening and gradual expansion of the outer skin by the hot liquid and the pull of the light weight of the lever.

From upwards of seventy experiments, every degree of effect was, obtained from zero to a more or less definite maximum contraction, as well as

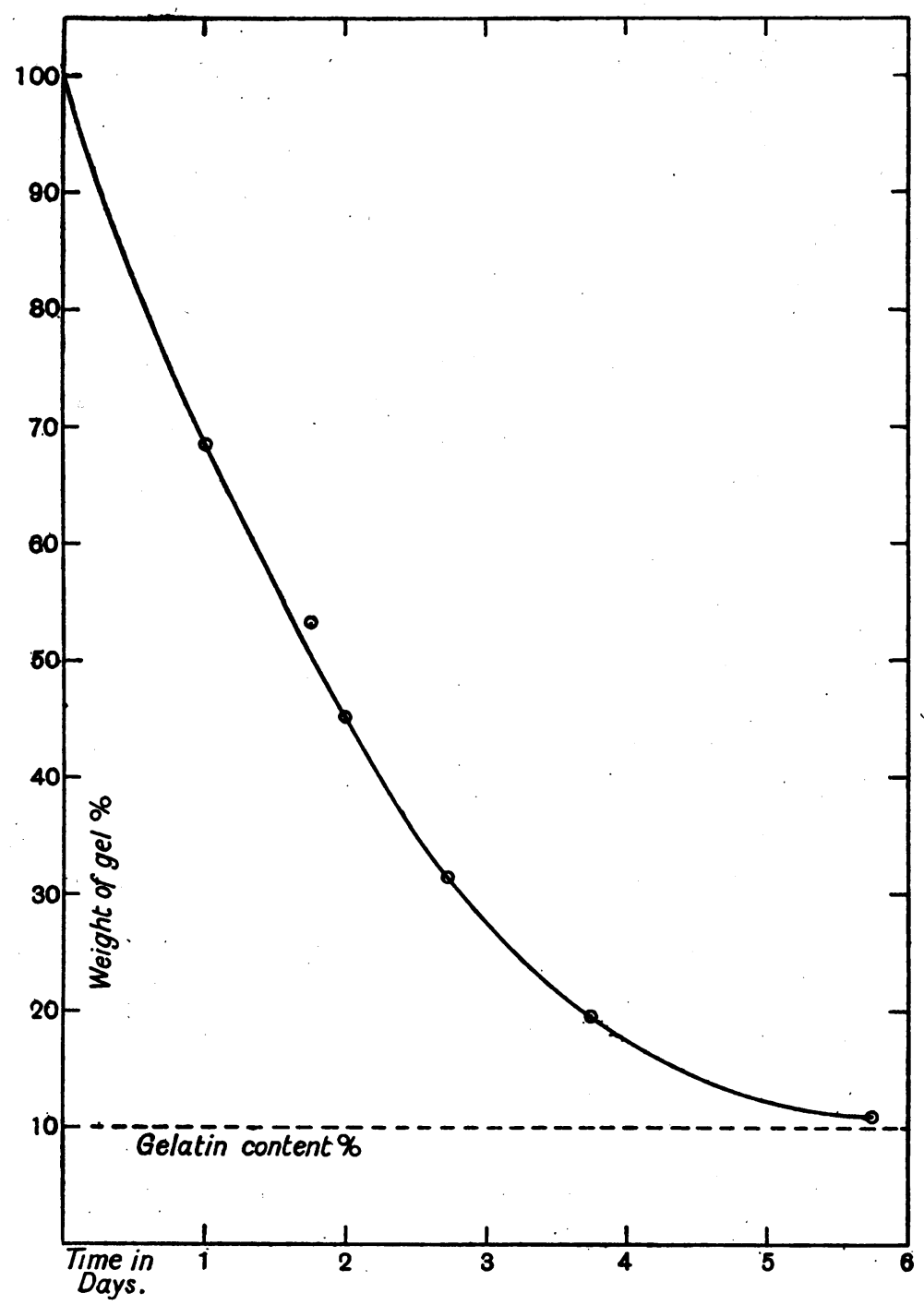

Fig. 2

indefinite expansion until melting occurred, according to the state of the outer skin of the gel. Usually very small contractions were observed on the first day, but after leaving the gel mounted in the empty apparatus over 
night, about $16 \mathrm{hrs.,} \mathrm{a} \mathrm{much} \mathrm{greater} \mathrm{effect} \mathrm{was} \mathrm{usually} \mathrm{obtained.} \mathrm{Keeping}$ the gels in oil prevented any increase in contraction with time, whether before or after the first measurement. A second heating immediately following the first gave much less, or no contraction; a third consecutive heating invariably none. Keeping for 16-24 hrs. however usually restored or increased the effect. If the heating was continued too long, the outer skin melted before the interior of the gel and rapid expansion immediately occurred. That the contraction in length was due to an actual expansion in volume was proved by the facts that cylinders of less height than diameter increased in height on heating, and that gels confined in test-tulves, so that increase of diameter was prevented, also showed a linear expansion.

On account of its lack of adhesiveness, there was no difficulty in moulding cylinders of agar gel. These were prepared of 2.5 per cent. agar. They came out of the small glass tubes without any injury, but always showed immediate expansion in length when heated.

The results of a selection of the experiments are tabulated below. The specific linear contractions are given, as well as the calculated specific volume expansions, for comparison with von Bjerkén's data. No. 5 was a cylinder with height greater than its diameter. The times are measured from the moment of painting. The dimensions are given in $\mathrm{cm}$.

The results appear to indicate, either that the actual thermal expansion of fresh gelatin gels is greater than that of the contained liquid, or that it becomes so with time. A number of experiments by various methods were made to determine this effect. Unexpected difficulties were encountered in obtaining concordant results, and when such had been obtained by one method, the values were found to differ greatly from an equally concordant set resulting from a different process. The question requires further study, and it is proposed to investigate the densities and coefficients of expansion of pure dry gelatin and agar and of a series of gels and sols of different concentration and age. This will also determine the total contraction of gel and water on swelling.

The Heat of Imbibition of Gelatin.

The heat of imbibition of air-dry sheet gelatin was found by Wiedemann and Lüdeking [1885] from three experiments as $6 \cdot 2,5 \cdot 3$ and $5 \cdot 6$ cal. giving a mean value of 5.7. The same observers obtained the value -3.7 as the heat of solation of $1 \mathrm{~g}$. of gelatin.

The gelatin for the present experiment was dried in the air oven at $110^{\circ}$ 


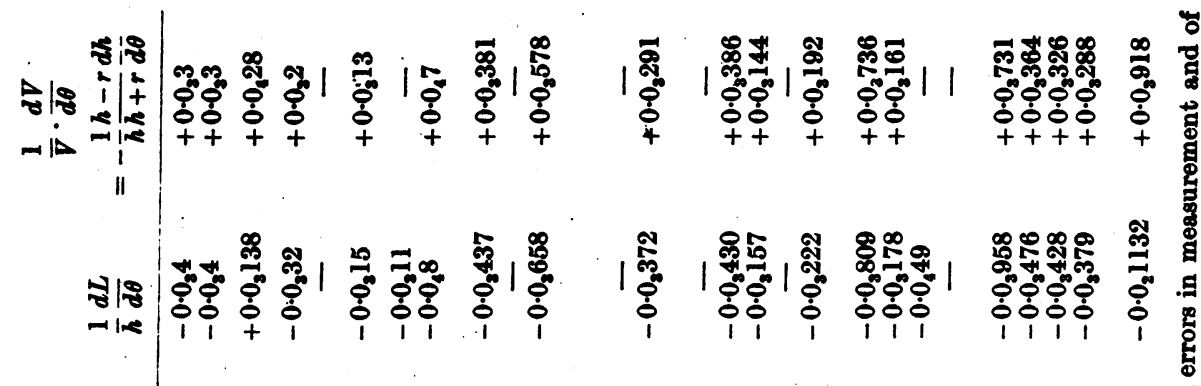

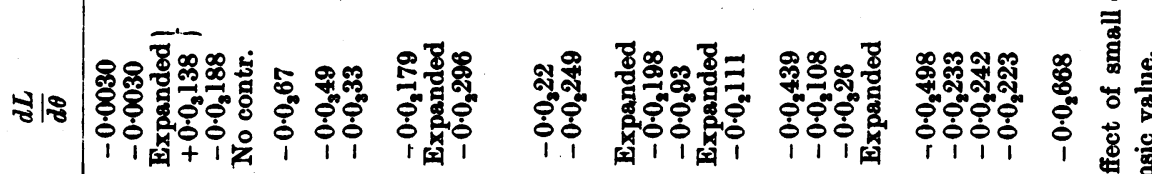

11

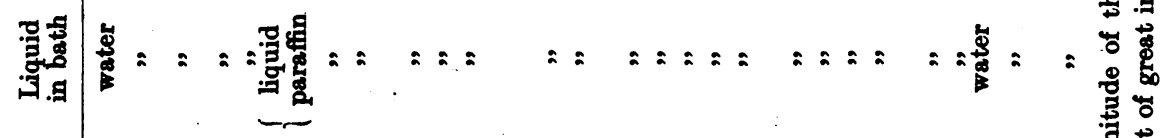

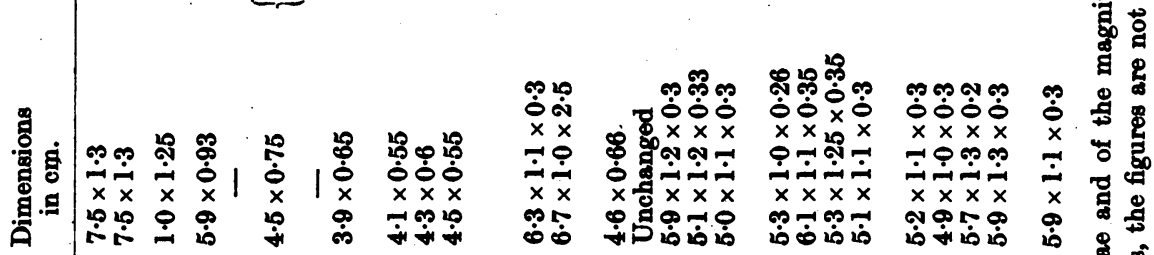

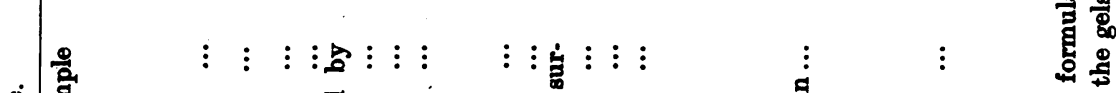

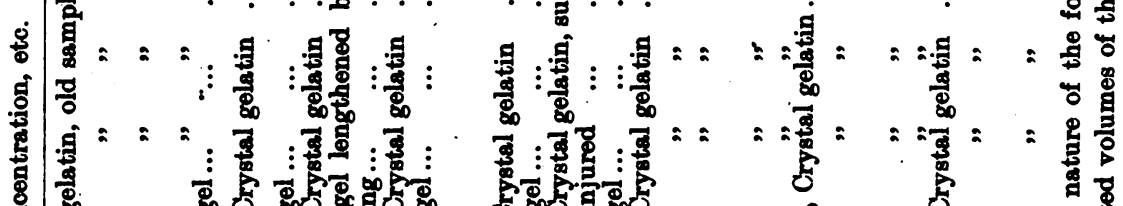

ठํㅇ

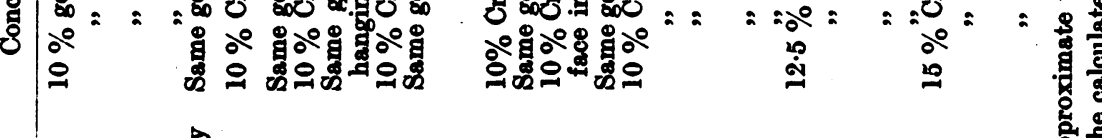

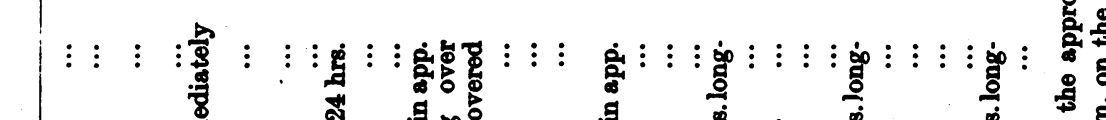

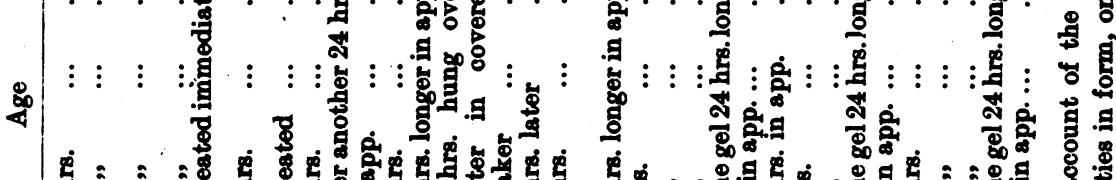

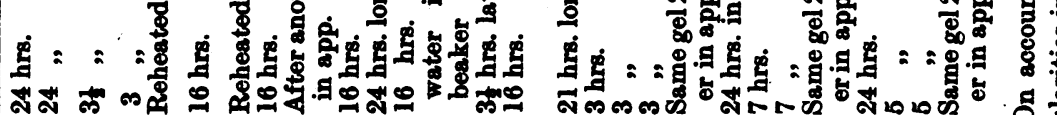
它 
for about 24 hours until constant in weight. The loss corresponded to $15 \cdot 70$ per cent. of moisture.

A small Dewar vacuum flask, holding about 150 cc., was used as calorimeter and was provided with a mechanical glass stirrer so that the rate of cooling was nearly constant. The temperatures were indicated by a Beckmann thermometer. The change of temperature by radiation per degree difference was only $0.0015^{\circ}$ per 15 seconds. Practically the whole of the heat was evolved within about four minutes. The results were corrected by Regnault's method. Two determinations gave:

\begin{tabular}{|c|c|c|c|c|c|}
\hline \multirow{3}{*}{$\begin{array}{l}\text { Gelatin taken ... } \ldots \\
\text { Total water equivalent }\end{array}$} & & & & (1) & (2) \\
\hline & $\ldots$ & $\ldots$ & $\cdots$ & 3.942 & 2.083 \\
\hline & $\ldots$ & $\therefore$ & $\ldots$ & $147 \cdot 841$ & $139 \cdot 845$ \\
\hline Initial temperature of gela & & ... & $\ldots$ & $21 \cdot 2^{\circ}$ & $19 \cdot 2^{\circ}$ \\
\hline Initial temperature of calo & rimeter & $\ldots$ & $\ldots$ & $20 \cdot 500^{\circ}$ & $18 \cdot 486^{\circ}$ \\
\hline Final temperature $\quad$... & $\ldots$ & $\ldots$ & $\because .$. & $21.413^{\circ}$ & $18.977^{\circ}$ \\
\hline Rise in temperature ... & $\ldots$ & $\ldots$ & $\ldots$. & $0.913^{\circ}$ & $0.491^{\circ}$ \\
\hline Corresponding heat value & $\ldots$ & $\ldots$ & $\ldots$ & 132.368 oal. & $68.664 \mathrm{cal}$. \\
\hline Heat added with gelatin & $\ldots$ & $\ldots$ & $\ldots$ & 1.242 & 0.656, \\
\hline $\begin{array}{l}\text { Heat of swelling per g. of } \\
\text { Heat absorbed by solation }\end{array}$ & $\begin{array}{l}\text { Ary gela } \\
\text { of gelati }\end{array}$ & $\begin{array}{l}\text { tin } \\
\text { in per } g\end{array}$ & $\ldots$ & $\begin{aligned} 33.264 & \\
0.177 & \end{aligned}$ & $\begin{array}{rl}32.649 & " \prime \\
0.304 & \sharp\end{array}$ \\
\hline Corrected heat of imbibitio & & $\ldots$ & $\ldots$ & $33.541 "$ & 32.953 \\
\hline Mean ... & $\ldots$ & $\ldots$ & $\ldots$ & 一 & $33 \cdot 25$ \\
\hline
\end{tabular}

The actual heat of swelling is greater than the observed value, in consequence of the absorption of heat by the solation of some of the gelatin by the water in the calorimeter. This is proportional to the mass of water used. The exact amount solated during the time of the experiment is however unknown. An experiment showed that $100 \mathrm{~g}$. of water are capable of taking up $0.114 \mathrm{~g}$. of solid matter from sheet gelatin at $20.2^{\circ}$ during the time of the experiment, which corresponds to $0 \cdot 135 \mathrm{~g}$. of air-dry'gelatin. Using. this value and Wiedemann and Luideking's figure for the heat of solation, the quantities of heat absorbed are 0.177 cal. and 0.304 cal. per gram respectively.

The values obtained do not agree very well. This may be due to the great influence of the previous thermal history of the gelatin upon its structure. It will be noticed that Wiedemann and Lüdeking's results showed a similar variation.

The writer wishes to express his indebtedness to Prof. J. C. Philip for the kind loan of the calorimetric apparatus. 


\section{SUMmary.}

The low diffusion constant of the natural emulsoids, by retarding the crystallisation of the hot sols, allows the accumulation of a large excess concentration, which, together with their large value of $K$ in von Weimarn's formula, causes gelation on cooling. The small crystallisation velocity, due to this low diffusion constant, is also responsible for the permanency of the gels and the hysteresis of the sols, since it prevents subsequent development of larger crystals in gels and retards changes in the aggregation of sol particles.

The viscosity changes in the sols of the natural emulsoids, by heating, are seen to be in conformity with Einstein's formula, since thermal changes cause alterations in the numbers, as well as the size, of the crystallisation centres.

The application of von Weimarn's theory to the reversible sol-gel transformation implies that gels should comprise two phases: an ultramicroscopic solid phase, bathed in a liquid dispersion medium, from which it has crystallised, and which it retains partly by molecular and partly, by capillary forces.

Consideration of the conditions of occurrence of spherites shows that complex organic substances and such as are highly aggregated in solution tend to crystallise in this form, and this leads to the supposition that the ultimate solid particles of gels are spherites. This is in conformity with the ultramicroscopic appearance, and receives further support from the fact that, by suitable means, gelatin can be made to assume the form of microscopic spheres.

The specific surface of gels is very large, and consequently the volume of liquid adsorbed at the liquid-solid boundary surfaces is appreciable. This liquid is under an internal pressure intermediate between those of the solid and of the liquid. This intermediate value of the internal pressure is much greater than that of the free liquid and conditions certain peculiarities in the properties of gels.

The theory forms a basis for the coordination of the properties of gels, and those predicted for such disperse systems are in accordance with the phenomena exhibited by gels. The experimental data at present available are insufficient for a complete mathematical discussion of the theory. Where the previous records are at variance with the theory, the data have been found to be in error. 


\section{REFERENCES.}

Amagat (1893). Ann. chim. phys. [vi], 29, 505. Anderson (1914). Zeitsch. physikal. Chem. 88, 191. Arisz (1915). Koll. chem. Beihefie, 7, 1.

Bachmann (1911). Zeitoch. anorg. Chem. 78, 150. (1917). Zeitsch. anorg. Chem. 100, 1.

Bemmelen (1878). Ber. 11, 2232. (1880). Ber. 13, 1466.

Bjerkén (1891). Ann. Phys. Chem. 48, 819.

Bradford (1916, 1). Science Prog. 10, 369.

- (1916, 2). Biochem. J. 10, 169. $(1917,1)$. Biochem. J. 11, 14. (1917, 2). Science Prog. 12; 63.

Brewster (1853). Trans. Roy. Soc. Edin. 20, 607.

Bütschli (1892). Untersuchungen uber mikroskopische Schaüme und das Protoplasma, Leipzig.

- (1896). Uber den Bau quellbarer Körper, Göttingen.

(1898). Untersuchungen uber Strukturen, Leipzig.

(1900). Untersuchungen iber den Mikrostruktur kïnstlicher u. natürlicher Kieseloäure-gallerten, Hęidelberg.

Dafert (1887). Ber. bot. Ges. 5, 113.

Dallas (1875). Proc. Roy. Soc. Edin. 9, 129.

Fischer (1902). Beitr. z. Biol. der Pflanzen, 8, 76.

Frankenheim (1835). Die Lehre von der Kohäsion, Breslau. (1860). Ann. Phys. Chem. 111, 1.

Garrett (1903). Disertation, 51, Heidelberg.

Gerike (1915). Koll. Zeitsch. 17, 78.

Habermann (1879). Annalen, 197, 113.

Hardy (1900). Proc. Roy. Soc. 65, 95.

Hatechek (1912). Koll. Zeitsch. 11, 158. (1914). Koll. Zeitsch. 15, 226. (1917). Trans. Farad. Soc. 12, 17.

Katz (1917). Koll. chem. Beihefte, 9, 1.

Leitgeb (1888). Mitt. bot. Inet. Gratz, 2, 257.

Levites (1908). Koll. Zeitsch. 2, 162, 240.

Lieck (1904). Ann. Phys. Chem. iv, 14, 149.

Lillie (1907). Amer. J. Physiol. 20, 127.

Linok (1839). Ann. Phys. Chem. 46, 258.

Linnemann (1874). Annaten, 171, 293.

Lüdeking (1888). Ann. Phys. Chem. 85, 552.

Madan (1901). J. Chem. Soc. 79, 922.

Masson (1891). Nature, 48, 345.

Moeller (1916). Koll. Zeitsch. 19, 205. (1917). Koll. Zeitoch. 20, 257.

Monnier (1874). Compt. Rend. 78, 300.

Moore (1915). Proc. Roy. Boc. B. 89, 27.

Nägeli (1858). Pflanzenphysiologioche Untersuchungen, Heft 2. Die Stärkekörner, Zürich, 332.

$(1862,1)$. Sitzungsber. K. Akad. Munchen, 1, 311. $(1862,2)$. Sitzungeber. $K$. Akad. Munchen, $1,316$.

Ostwald (1891). Lehrbuch, 1, 1039. (1897). Zeitsch. physikal. Chem. 82, 289.

Pauli (1902). Der kolloidale Zustand und die Vorgänge in der lebendigen Substanz, Braunochweig. 
Pauli (1913). Beiträge, 8, 225.

Pauli and Rona (1902). Beiträge, 2, 4.

Prootor (1914). J Chem. Soc. 105, 313.

Rainey (1858). On the mode of formation of shells, bone, etc. London.

Reinke (1879). Bot. Abhandl. (Hanstein), 4, 1.

Richards and Archibald (1901). Aimer. Chem. J. 26, 61.

Rodewald (1897). Zeitoch. physikal. Chem. 24, 193.

Rohloff (1007). Physikal. Zeitsch. 8, 442.

Schroeder (1803). Zeitoch. physikal. Chem. 45, 109.

Syniewski (1802). Annalen, 824, 206.

Vesque (1874). Compt. Rend. 78, 149.

Vogelsang (1875). Die Krystalliten, Bonn.

Weimarn (1910). J. Russ, Phys. Chem. Soc. 42, 653. (1914). Zur Lehre von den Zuständen der Materie, Dresden.

Wiedmann and Lüdeking (1885). Anr. Phys. Chem. 25, 147.

Zsigmondy (1911). Zeitsch. anorg. Chem. 71, 356.

Zsigmondy and Bachmann (1912). Koll. Zeitoch. 11, 145. 